\title{
Parametric study of lateral stability for a railway vehicle ${ }^{\dagger}$ \\ Joon-Hyuk Park ${ }^{*}$, Hyo-In Koh and Nam-Po Kim \\ Korea Railroad Research Institute, Uiwang, 360-1, Korea
}

(Manuscript Received July 20, 2010; Revised March 3, 2011; Accepted April 14, 2011)

\begin{abstract}
This paper deals with the lateral stability for a railway vehicle. Particularly, nonlinear critical speed is investigated via bifurcation analysis. Shooting method and trust region algorithm are applied to calculate the limit cycle of the nonlinear dynamic vehicle model. In case of the investigated vehicle model, the influences of the suspension parameters on the critical speeds are not large. The effects of friction coefficient are also small. However, there is an overall consistency that the nonlinear critical speed is decreased when the stiffness of the suspension is reduced. Study of the excitation conditions where the stable limit cycles occur gives very useful information to understand the nonlinear dynamic behavior of a railway vehicle and to test the vehicle with roller rig test stands. According to the results, the direction of the excitation is very important as well as the amplitude and frequency to acquire accurate nonlinear critical speed.
\end{abstract}

Keywords: Railway vehicle; Stability; Bifurcation; Nonlinear critical speed; Excitation conditions

\section{Introduction}

The stability analysis of railway vehicles is one of the classical research areas that takes on increasing importance with the development of high speed railway vehicles. Particularly, hunting stability is the most significant problem in dynamic systems of railway vehicles. Here, "hunting" means the dynamic combination of two (lateral and yaw) degrees of freedom when the behavior of a wheelset is unstable [1]

The conventional railway wheelset, which consists of two conical wheels mounted on a common axle, undergoes lateral oscillations from a small disturbance when it is rolling. If the rolling speed is low, these lateral oscillations converge to zero (track center). However, at a certain speed or faster, the railway wheelset loses its stability and shows unstable motion. This certain speed is called "critical speed". Therefore, in the railway vehicle, wheelsets are supported to the bogies or car bodies with high stiffness elements in order to increase the critical speed and the railway vehicle should be designed so that its critical speed can be largely higher than the operating speed. Hence, the hunting stability analysis and prediction of the critical speed has been very important in the design process of the railway vehicle, and various related researches have been reported [2-4].

The first concern was the linear stability analysis of the lin-

\footnotetext{
${ }^{\dagger}$ This paper was recommended for publication in revised form by Editor Yeon June Kang

*Corresponding author. Tel.: +82 31460 5276, Fax.: +82 314605299

E-mail address: jhpark74@krri.re.kr

(c) KSME \& Springer 2011
}

earized dynamic model of a railway vehicle. But, many research results have pointed out that nonlinear effects should be considered for the complete investigation of the hunting motion. Generally, because the nonlinear critical speed is lower than the linear critical speed, it can be said that nonlinear analysis is more important than linear analysis for the stability assessment of the railway vehicles. One of the remarkable methods to acquire the bifurcation diagram of the nonlinear railway vehicle system was suggested by $\mathrm{Ch}$. Kass-Petersen [5] and has been advanced by several researchers $[4,6]$. His idea was commercialized as so-called 'PATH', and a multibody-system (MBS) analysis software, SIMPACK includes the bifurcation analysis tool based on his method. Due to his efforts, bifurcation analysis has been widely used for nonlinear stability analysis of railway vehicles.

In recent years, related researches have been concentrated on the assessment of the stability under certain conditions. Zboninski et al. studied the nonlinear stability analysis of a rail vehicle on a curved track [7]. Zeng et al. investigated the effects of the coupler traction force on the nonlinear critical speed in straight and curved tracks [8]. Effects of the nonlinearities of the suspension system on the lateral stability of railway freight trucks have been one of the attractive research areas because freight trucks usually show chaotic motion due to nonlinear dry friction dampers $[9,10]$

Linear stability analysis is still useful for preliminary calculation during the vehicle design in the industry, particularly, when no complete information concerning the actual wheel/rail geometry is available in an early phase. Polach has studied the comparability of linearized and nonlinear stability assessment 
[11] and he recommended that conservative parameters should be used to secure some safety margin. He also introduced several methods of nonlinear stability analysis used in industrial applications and reported that careful choice of method was also important for a reliable stability prediction [12].

As stated above, many researchers have studied how to analyze the nonlinear dynamic behavior of a railway vehicle and estimated the nonlinear critical speed in certain environments. However, an investigation of the domains of attraction or condition for generating the limit cycle has been nearly ignored in the previous research, even though it can give significant information for the assessment of the stability, particularly, when using the stability test equipment such as roller rig test stands.

Thus, in this paper, the domains of transient conditions where the limit cycle occurs are investigated. In section 2, a dynamic model of a railway vehicle and wheel/rail contact model are introduced. Bifurcation analysis of the model is carried out in section 3, and the influences of some model parameters are also discussed. The domains of transient conditions to generate the limit cycle are described in section 4 . Finally, section 5 discusses the main conclusion and suggestions for future research.

\section{Dynamic model of a railway vehicle}

\subsection{Creepages and creep forces}

The dynamic behavior of a railway vehicle is significantly affected by the interactive forces between the wheel and rail. These forces depend on the adhesion, creep and wheel/rail geometry. In general, the difference of the circumferential velocity between two rolling bodies is defined as creepage. In railway engineering, creepages are usually determined as Ref. [13]

$$
\begin{aligned}
& \xi_{x}=\frac{\left(\dot{\mathrm{r}}_{\mathrm{p}}^{\mathrm{w}}-\dot{\mathrm{r}}_{\mathrm{p}}^{\mathrm{r}}\right) \cdot \mathbf{t}_{1}^{\mathrm{r}}}{V} \\
& \xi_{y}=\frac{\left(\dot{\mathrm{r}}_{\mathrm{p}}^{\mathrm{w}}-\dot{\mathrm{r}}_{\mathrm{p}}^{\mathrm{r}}\right) \cdot \mathbf{t}_{2}^{\mathrm{r}}}{V}, \\
& \xi_{s p}=\frac{\left(\boldsymbol{\omega}^{\mathrm{w}}-\boldsymbol{\omega}^{\mathrm{r}}\right) \cdot \mathbf{n}^{\mathrm{r}}}{V},
\end{aligned}
$$

where $\xi_{x}, \xi_{y}$, and $\xi_{s p}$ are longitudinal, lateral and spin creepages, respectively; $\dot{\mathbf{r}}_{\mathrm{p}}^{\mathrm{w}}$ and $\dot{\mathbf{r}}_{\mathrm{p}}^{\mathrm{r}}$ are the time derivatives for the contact position vector of wheel and rail in generalized coordinates, respectively; $\mathbf{t}_{1}^{\mathrm{r}}$ and $\mathbf{t}_{2}^{\mathrm{r}}$ are the unit orthogonal tangent vectors to the rail at the contact point in the longitudinal and lateral directions; $\mathbf{n}^{\mathrm{r}}$ is the normal vector unit to the surface at the contact point; and $V$ is the magnitude of the wheel velocity along the longitudinal tangent vector at the contact point.

When the coordinate system of the contact plane between wheels and rails is located as shown in Fig. 1, from Eqs. (1)(3), creepages are given as follows:

Left wheel:

$$
\xi_{x L}=\frac{V \cos \psi+\dot{y} \sin \psi-r_{L}\left(V / r_{0}+\dot{\psi} \sin \phi\right)-g_{a} \dot{\psi} \cos \phi}{V},
$$

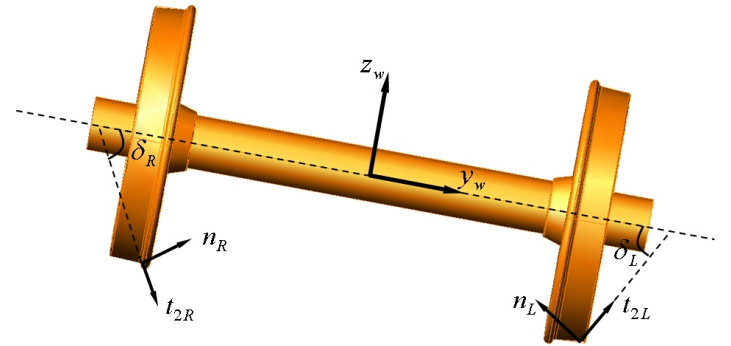

Fig. 1. Contact plane coordinates.

$$
\begin{aligned}
& \xi_{y L}=\frac{r_{L} \dot{\phi} \cos \delta_{L}+a \dot{\phi} \sin \delta_{L}-V \cos \left(\delta_{L}+\phi\right) \sin \psi+\dot{y} \cos \left(\delta_{L}+\phi\right) \cos \psi}{V}, \\
& \xi_{s p L}=\frac{-V / r_{0} \sin \delta_{L}+\dot{\psi} \cos \left(\delta_{L}+\phi\right)}{V},
\end{aligned}
$$

Right wheel:

$$
\begin{aligned}
& \xi_{x R}=\frac{V \cos \psi+\dot{y} \sin \psi-r_{R}\left(V / r_{0}+\dot{\psi} \sin \phi\right)+g_{a} \dot{\psi} \cos \phi}{V} \\
& \xi_{y R}=\frac{r_{R} \dot{\phi} \cos \delta_{R}+a \dot{\phi} \sin \delta_{R}-V \cos \left(\delta_{R}-\phi\right) \sin \psi+\dot{y} \cos \left(\delta_{R}-\phi\right) \cos \psi}{V} \\
& \xi_{s p R}=\frac{-V / r_{0} \sin \delta_{R}+\dot{\psi} \cos \left(\delta_{R}-\phi\right)}{V}
\end{aligned}
$$

Here, $g_{a}$ is a half of track gauge; $r_{0}, r_{R}, r_{L}$ are nominal rolling radius, rolling radii of right and left wheels, respectively; $\delta_{L}, \delta_{R}$ are contact angles of right and left wheels, respectively; $\dot{y}$ is the lateral velocity of a wheelset; $\psi, \dot{\psi}$ are yaw and yaw velocity; $\phi, \dot{\phi}$ are roll and roll velocity.

Creep forces are generated by the difference in strain rates of the two bodies and mainly depend on creepages, contact region and friction coefficient. Excellent theories to calculate the creep forces were developed by Kalker [14]. He suggested a linear theory for estimating linearized creep force in the very small creepage region, and a simplified theory for calculating nonlinear creep forces. He also developed a famous computing program called FASTSIM, and it has been applied to most of MBS S/W for railway vehicle dynamics. Polach [15] suggested a faster and a simpler algorithm than FASTSIM for the computation of wheel/rail force, which is based on Kalker's simplified theory. In this paper, Polach's algorithm is used for the calculation of creep forces. According to his algorithm, the longitudinal and lateral creep forces $F_{x}, F_{y}$ are calculated as

$$
\begin{aligned}
& F_{x}=-N_{0} \cdot M \cdot \xi_{x} / \xi_{c}, \\
& F_{y}=-N_{0}\left(M \cdot \xi_{y}+F_{y s} \cdot \xi_{\text {spin }}\right) / \xi_{c}, \\
& \xi_{c}=\left\{\begin{array}{l}
\sqrt{\xi_{x}^{2}+\left(\xi_{y}+a \cdot \xi_{\text {spin }}\right)^{2}}:\left(\xi_{y}+a \cdot \xi_{\text {spin }}>\xi_{y}\right) \\
\sqrt{\xi_{x}^{2}+\xi_{y}^{2}},\left(\xi_{y}+a \cdot \xi_{\text {spin }} \leq \xi_{y}\right)
\end{array},\right. \\
& M=\frac{2 \mu_{s}}{\pi} \cdot\left(\frac{\varepsilon}{1+\varepsilon^{2}}+a \tan (\varepsilon)\right), \\
& F_{y s}=9 a \mu_{f} K_{s}\left(\varepsilon_{m}\left(-\frac{\delta^{3}}{3}+\frac{\delta^{2}}{2}-\frac{1}{6}+\frac{1}{3}\left(1-\delta^{2}\right)\right)\right) \\
& K_{s}=1+6.3 \cdot\left(1-e^{a / b}\right), \\
& \delta=\frac{\varepsilon_{m}^{2}-1}{\varepsilon_{m}^{2}+1} .
\end{aligned}
$$




$$
\begin{gathered}
m_{w} \ddot{y}_{w j}+2 C_{p y} \dot{y}_{w j}+2 K_{p y} y_{w j}-2 K_{p y} y_{b i}-2 C_{p y} \dot{y}_{b i} \mp 2 K_{p y} \cdot l_{w b} \cdot \psi_{b i} \mp 2 C_{p y} \cdot l_{w b} \cdot \dot{\psi}_{b i}= \\
F_{y l j}+F_{y r j}+N_{y l j}+N_{y r j}, \\
I_{w z} \ddot{\psi}_{w j}+I_{w y}\left(\frac{r_{0}}{V}\right) \dot{\phi}_{w j}+K_{p x} l_{w a}^{2} \psi_{w j}+C_{p x} l_{w a}^{2} \dot{\psi}_{w j}-K_{p x} l_{w a}^{2} \psi_{b i}-C_{p x} l_{w a}^{2}= \\
R_{x r j}\left(F_{y r j}+N_{y r j}\right)-R_{y r j} F_{x r j}+R_{x l j}\left(F_{y l j}+N_{y l j}\right)-R_{y l j} F_{x l j}, \\
m_{b} \ddot{y}_{b i}+\left(4 C_{p y}+C_{s y}\right) \dot{y}_{b i}+2\left(2 K_{p y}+K_{s y}\right) y_{b i}-2 C_{p y}\left(\dot{y}_{w j}+\dot{y}_{w(j+1)}\right) \\
-2 K_{p y}\left(y_{w j}+y_{w(j+1)}\right)-C_{s y} \dot{y}_{v}-2 K_{s y} y_{v} \mp l_{b b}\left(2 K_{s y} \psi_{v}+C_{s y} \dot{\psi}_{v}\right)=0, \\
I_{b z} \ddot{\psi}_{b i}+\left(2\left(2 C_{p y} l_{w b}^{2}+2 C_{p x} l_{w a}^{2}\right)+2 C_{s x} l_{b a}^{2}\right) \dot{\psi}_{b i}-2 C_{s x} l_{b a}^{2} \dot{\psi}_{v}-2 K_{s x} l_{b a}^{2} \psi_{v}+ \\
\left(2\left(2 K_{p y} l_{w b}^{2}+2 K_{p l} l_{w a}^{2}\right)+2 K_{s x} l_{b a}^{2}\right) \psi_{b i}-2 C_{p y} l_{w b} y_{w j}-2 K_{p y} l_{w b} y_{w j}-2 C_{p x} l_{w a}^{2} \psi_{w j}- \\
2 K_{p x} l_{w a}^{2} \psi_{w j}+2 C_{p y} l_{w b} \dot{y}_{w(j+1)}+2 K_{p y} l_{w b} y_{w(j+1)}-2 C_{p x} l_{w a}^{2} \dot{\psi}_{w(j+1)}-2 K_{p x} l_{w a}^{2} \psi_{w(j+1)}=0, \\
m_{v} \ddot{y}_{v}+C_{s y}\left(2 \dot{y}_{v}-\dot{y}_{b i}-\dot{y}_{b(i+1)}\right)+2 K_{s y}\left(2 y_{v}-y_{b i}-y_{b(i+1)}\right)=0, \\
I_{v} \ddot{\psi}_{v}+2 l_{b b}^{2}\left(C_{s y} \dot{\psi}_{v}+2 K_{s y} \psi\right)+4\left(C_{s x} l_{b a}^{2} \dot{\psi}_{v}+K_{s x} l_{b a}^{2} \psi_{v}\right)-2 C_{s x} l_{b a}^{2}\left(\dot{\psi}_{b i}+\dot{\psi}_{b(i+1)}\right) \\
-l_{b b}\left(C_{s y}\left(\dot{y}_{b i}-\dot{y}_{b(i+1)}\right)+2 K_{s y}\left(y_{b i}-y_{b(i+1)}\right)\right)-2 K_{s x} l_{b a}^{2}\left(\psi_{b i}+\psi_{b(i+1)}\right)=0,
\end{gathered}
$$

for, $j=1,2(i=1) ; j=3,4(i=2)$.

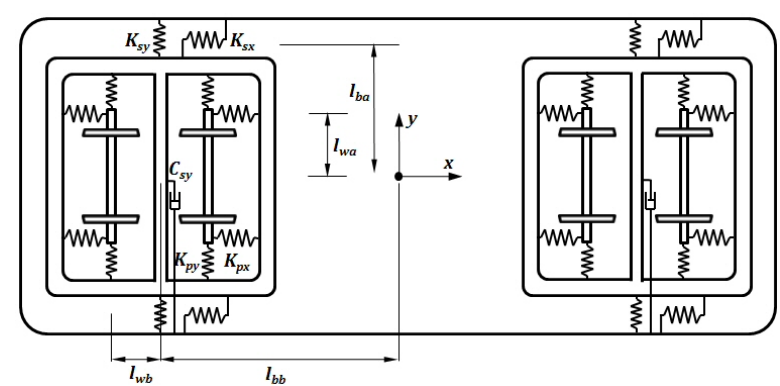

Fig. 2. Schematic diagram of a railway vehicle model.

Here, $\varepsilon$ and $\varepsilon_{m}$ are the gradient of tangential stress in the area of adhesion, $N_{0}$ is the normal force to the contact plane.

\subsection{Equations of motions for lateral stability analysis}

A dynamic model is established to analyze the lateral stability of a railway vehicle. It is assumed as a 14-D.O.F. four-axle vehicle composed of a car-body and two bogies including two wheelsets (Fig. 2). Each component is allowed to move in the lateral and yaw directions. Here, reference coordinates are located on the track center line and the vehicle travels along the positive $x$ direction.

Modeling of a railway vehicle has been well studied [13, 16, 17]; therefore, we briefly introduce the results. From the Newtonian method of dynamics, equations of motions of the model can be expressed as Eqs. (17)-(22).

Here, $y_{w j}$ and $\psi_{w j}$ are the lateral displacement and the attack angle of the jth wheelset, respectively; $y_{b i}$ and $\psi_{b i}$ are the lateral displacement and the attack angle of the ith bogie, respectively; Also, $y_{v}$ and $\psi_{v}$ denote the lateral displacement and the attack angle of the car body. The parameter values and the other physical parameters are defined in the Nomenclautre and Appendix.

If we use Kalker's linear theory and neglect higher-order terms, Eqs. (17) and (18) can be simplified to Eqs. (23) and (24) [17].

$$
\begin{aligned}
& m_{w} \ddot{y}_{w j}+2 C_{p y} \dot{y}_{w j}+2 K_{p y} y_{w j}-2 K_{p y} y_{b i}-2 C_{p y} \dot{y}_{b i} \mp \\
& 2 K_{p y} \cdot l_{w b} \cdot \psi_{b i} \mp 2 C_{p y} \cdot l_{w b} \cdot \dot{\psi}_{b i}=-\frac{2 f_{11}}{V}\left(\dot{y}_{w j}+\right. \\
& \left.\frac{r_{0} \lambda}{g_{a}} \dot{y}_{w j}-V \psi_{j w}\right)-\frac{2 f_{12}}{V} \dot{\psi}_{w j}-\frac{w_{A} \lambda}{g_{a}} y_{w j}, \\
& \quad I_{w z} \ddot{\psi}_{w j}+I_{w y} \frac{V}{r_{0}} \frac{\lambda}{g_{a}} \dot{y}_{w j}+K_{p x} l_{w a}^{2} \psi_{w j}+C_{p x} l_{w a}^{2} \dot{\psi}_{w j}- \\
& \quad K_{p x} l_{w a}^{2} \psi_{b i}-C_{p x} l_{w a}^{2}=-\frac{2 g_{a} f_{33} \lambda}{r_{0}} y_{w j}+\frac{2 f_{12}}{V}\left(\dot{y}_{w j}+\right. \\
& \left.\frac{r_{0} \lambda}{g_{a}} \dot{y}_{w j}-V \psi_{w j}\right)-2 g_{a}^{2} f_{33} \frac{\dot{\psi}_{w j}}{V}+g_{a} W_{A} \lambda \psi_{w j}-f_{22} \frac{\dot{\psi}_{w j}}{V},
\end{aligned}
$$

where $f_{11}, f_{12}, f_{22}$ and $f_{33}$ are the creep coefficients of Kalker; $\lambda$ is the equivalent conicity of the wheel; $W_{A}$ is the axle-load.

As stated earlier, the critical speed can be changed according to the wheel/rail geometry even though other parameters are almost the same [18]. Therefore, it is very important for the stability assessment. We used conventional Korean wheel and rail profiles. The wheel has $1 / 20$ conicity in the tread of wheel with $0.43 \mathrm{~m}$ nominal rolling radius. The head profile of the rail is Korean $60 \mathrm{~kg}$ rail with 1/40 inclination. Fig. 3 shows the contact characteristics of the applied wheel and rail.

\section{Nonlinear stability analysis}

\subsection{Bifurcation analysis}

It is known that limit cycles or periodic orbits bifurcate from the system equilibrium points via Hopf bifurcation. There are various methods to calculate the limit cycles such as center manifold theory, averaging, direct numerical integration, shooting method and so on. We used the shooting method [19] to investigate the limit cycle of the given railway vehicle by solving the boundary value problem.

The equations of motions, Eqs. (17)-(22), can be rewritten as the following form:

$$
\dot{X}=F(X, V) \text {. }
$$




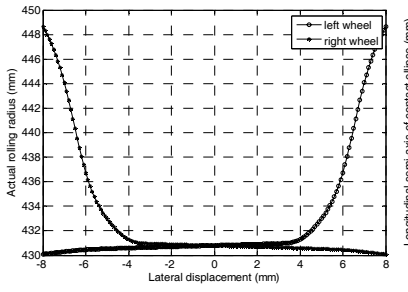

(a)

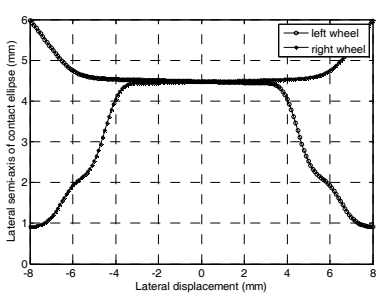

(c) (b)

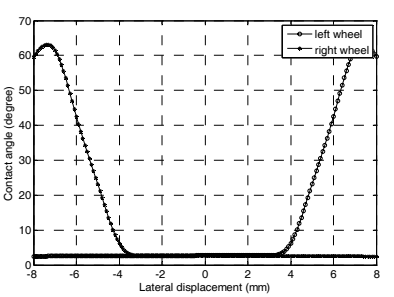

(d)

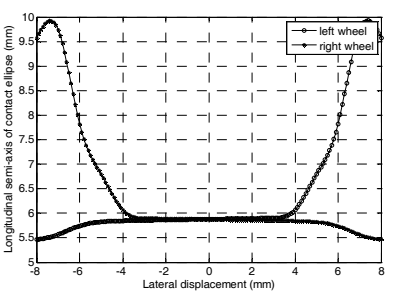

Fig. 3. Contact characteristics of the wheel and rail (a) actual rolling radius; (b) longitudinal semi-axis of contact ellipse; (c) lateral semiaxis of contact ellipse; (d) contact angle.

If the differential equation, Eq. (25) has the periodic solutions, the Poincaré map (section) can be used to find the periodic solutions. Assuming that the solutions, $\mathrm{X}$ at time $t=0$ are the periodic solutions with period, $\mathrm{T}$, the trajectories of $\mathrm{X}$ will hit the same points on the Poincaré map at $t=T$. Thus, we have:

$$
X(t)=X(t+T)
$$

Let $t$ be $t=T z$ in Eq. (25), following differential equation and its boundary condition can be obtained.

$$
\begin{aligned}
& \frac{d X}{d z}=T F(X, V), \\
& X(0)=X(1) .
\end{aligned}
$$

Therefore, if the proper Poincaré section can be constructed, Eq. (27) can be solved using the shooting method with the boundary condition, Eq. (28).

Using the fact that all state variables of Eq. (25) have the same period $\mathrm{T}$ with different amplitudes if one of the state variables has the period $\mathrm{T}$, the Poincaré section can be defined as

$$
\Sigma:=\left\{X \mid X_{1}>0, X_{2}=0\right\}
$$

where $X_{1}$ and $X_{2}$ denote the lateral displacement and velocity of the first wheelset, respectively. Let us consider the initial guesses of $X_{1}$ and $X_{2}$ to be arbitrary value and zero, respectively, the nonlinear algebraic equation can be obtained from Eqs. (27) and (28) with 28 unknown variables, which are $X_{n}(\mathrm{n}=3 \cdots 28), T$ and $V$. Thus, the algebraic equation can be solved by some iteration methods. In this study, the thrust region method is used to find the solution of the algebraic equation because it is robust and can be applied to illconditioned problems [20].

Since the trust region method is a local optimization algorithm, the choice of the initial guesses for obtaining reasonable solutions is very important. The first initial guesses should be a very small value for $X_{1}$, zeros for $X_{2 \ldots 28}$, and $V_{0}, T_{0}$, which are the velocity and period at the equilibrium state. $V_{0}$, $T_{0}$ can be found from the eigenvalue problem of the linearized equation for Eq. (25). The linearized state equations at equilibrium points can be expressed as

$$
\dot{X}=\left.\frac{\partial F_{i}}{\partial x_{j}}\right|_{x_{j}=0} X(i, j=1, \cdots 28) .
$$

$V_{0}$ is determined when one pair of complex conjugate eigenvalues of Eq. (30) reach the imaginary axis with increasing velocity, and $T_{0}$ is $2 \pi / \omega$, where $\omega$ is the absolute value of the eigenvalue.

\subsection{Stability of limit cycles}

The limit cycles can be classified into two: stable limit cycles and unstable limit cycles. If the state values are near the stable limit cycles, they will converge to the stable limit cycles. However, when they are close to the unstable limit cycles, finally they will go to the stationary points or other stable limit cycles. Stability of the limit cycles can be tested using the linearized state equation of Eq. (27), similarly to Eq. (30). The linearized equation at the limit cycle points is defined as

$$
\frac{d X}{d z}=\left.T \frac{\partial F_{i}}{\partial x_{j}}\right|_{x_{j}=X(1), V} X(i, j=1, \cdots 28) .
$$

If one pair of eigenvalues of Eq. (31) has zero or positive real values, the limit cycle at the velocity, $V$ can be determined to be unstable.

\subsection{Numerical results}

\subsubsection{Linearized model and linear Kalker model}

As stated in 3.1, bifurcation analysis is started from the initial guesses near the equilibrium points. Hence, the velocity and period of the equilibrium state have to be precalculated before the bifurcation analysis. The equilibrium state velocity, the so-called linear critical speed, has been often obtained from the linear simple model using Eqs. (19)-(24) where linear Kalker theory is applied. Table 1 shows the comparison results of the linear critical speeds between the linearized model and the linear Kalker model. The linear critical speeds of the linear Kalker model are slightly lower than those of the linearized model, and the differences are about $6 \sim 10 \%$. The variations of the linear critical speeds according to suspension parameters are not large in both models, differently from our expectations.

The linear Kalker model can be used for rough design of a railway vehicle as well as the linearized model, but the linear 
Table 1. Analysis results of the linear critical speed.

\begin{tabular}{c|c|c|c}
\hline & $V_{0 k}(\mathrm{~m} / \mathrm{s})$ & $V_{0}(\mathrm{~m} / \mathrm{s})$ & $\frac{V_{0 k}}{V_{0}}(\%)$ \\
\hline Nominal values (Case 1) & 93.513 & 100.723 & 92.84 \\
60\% Kpx (Case 2) & 90.21 & 99.932 & 90.27 \\
140\% Kpx (Case 3) & 90.741 & 96.163 & 94.36 \\
60\% Kpy (Case 4) & 91.043 & 97.778 & 93.11 \\
140\% Kpy (Case 5) & 93.990 & 100.379 & 93.64 \\
$60 \%$ Ksy (Case 6) & 91.894 & 98.823 & 92.99 \\
140\%Ksy (Case 7) & 95.204 & 102.688 & 92.71 \\
60\%Csy (Case 8) & 91.484 & 97.132 & 94.19 \\
140\%Csy (Case 9) & 92.164 & 101.155 & 91.11 \\
\hline
\end{tabular}

Table 2. Critical speeds of the half model and full model.

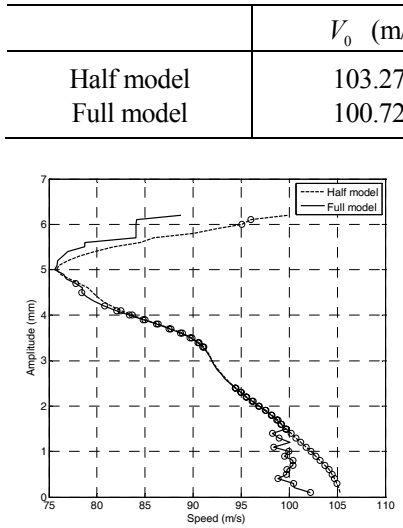

(a)

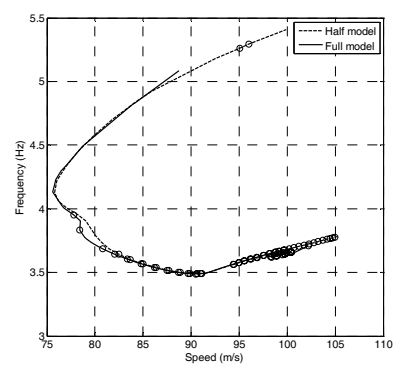

(b)
Fig. 4. Bifurcation analysis results of the full model and half model (a) bifurcation diagram; (b) frequency diagram.

critical speed of the linearized model is applied for the starting point of bifurcation analysis in this paper.

\subsubsection{Full model and a half model}

To reduce the time cost for numerical analysis, one bogie model had been applied instead of a vehicle model [16, 21]. However, it is rarely used except some special cases because the stability of a bogie cannot represent that of a vehicle. On the other hand, a half model composed of a half car body and a bogie with two wheelsets is often used to study the running performance of a railway vehicle on curve track [22, 23] because a half model and a full model are similar in low frequency response such as response on curve track.

In this section, the lateral stability of a half model is analyzed to determine whether the half model is suitable for stability analysis. Because yaw motion of a half car body is ignored in the half model, it is a 7-D.O.F. system. Fig. 4 shows the bifurcation diagram of the full model and half model. The results are summarized at Table 2 .

As shown in the figure, there is a good agreement between their nonlinear critical speeds even though the linear critical speed of the half model is a bit higher than that of the full model. Here, the nonlinear critical speed denotes the lowest speed in the bifurcation diagram. Hunting frequency of the half model is also similar to that of the full model. However, some different aspects between them are shown in the high and low amplitude regions. In case of the full model, the amplitude of the limit cycle suddenly jumped up at about 78.7 and $84 \mathrm{~m} / \mathrm{s}$ in the high amplitude region. As a result, it reaches the maximum amplitude (limit amplitude of this analysis, 6.2 $\mathrm{mm}$ ) at relatively low speed compared to the half model. Also, it shows some complex shape in the low amplitude region. On the other hand, the limit cycle of the half model has a sharper and smoother curve in the high amplitude region and has the maximum amplitude at high speed. It also displays a smooth curve in the low amplitude region. In spite of some differences between the local shapes of bifurcation diagrams of both models, the half model can be considered to reduce the computation time for predicting the nonlinear critical speed.

In Fig. 4, solid and dashed lines denote the stable limit cycles, and lines with symbols ( $\bullet$ and $-\odot-)$ represent the unstable limit cycles. As stated earlier, if the state values are close to the unstable limit cycles, they will move to the stationary points or stable limit cycles. For example, if the states are closely located under the unstable limit cycles when the vehicle speed is between the nonlinear critical speed and linear critical speed, they will go to the stationary point, zero. They will move up to the stable limit cycles when they are over the unstable limit cycles. However, when the speed is higher than the linear critical speed, the states always have limit cycles if their initial values are not zero and they always converge to zero when the speed is lower than the nonlinear critical speed.

\subsubsection{Primary and secondary suspension parameters}

Suspension parameters are important factors for determining the critical speed as well as the wheel/rail geometry. Generally, low stiffness of the primary suspension causes a low critical speed and high stiffness increases the critical speed. However, those suspension effects are changed by the wheel/rail geometry. In other words, at a certain wheel/rail geometry, low stiffness suspension gives us the high critical speed or vice versa. Therefore, suspension properties should be carefully designed with considering wheel/rail geometry.

In this section, for the given wheel/rail geometry, the suspension effect on the nonlinear critical speed is analyzed. For this work, each suspension parameter is assumed to be $40 \%$ lower or higher than the nominal value and we compare the results of bifurcation analysis for each case defined in Table 1 .

Fig. 5 shows the bifurcation diagram according to the change of the primary suspension stiffness. The linear critical speed has the highest value when the longitudinal stiffness of the primary suspension is $6.6 \mathrm{MN} / \mathrm{m}$ (nominal value). Particularly, the linear critical speed of Case 3 is lower than Cases 1 and 2. For the nonlinear critical speed, Case 3 is slightly higher than Case 1. But, the increase is smaller than expected, compared to the increase of stiffness. This implies that the nominal stiffness of longitudinal direction in the primary sus- 


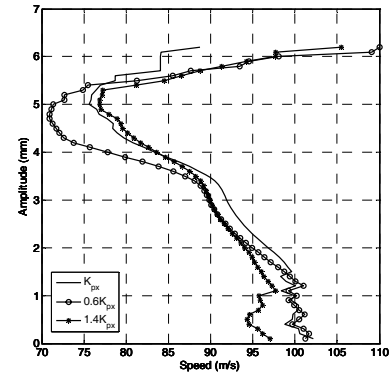

(a)

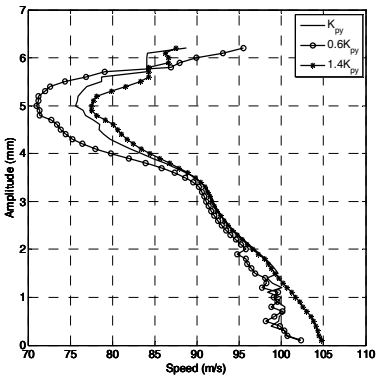

(b)
Fig. 5. Bifurcation analysis results along the change of primary suspension (a) longitudinal stiffness; (b) lateral stiffness.

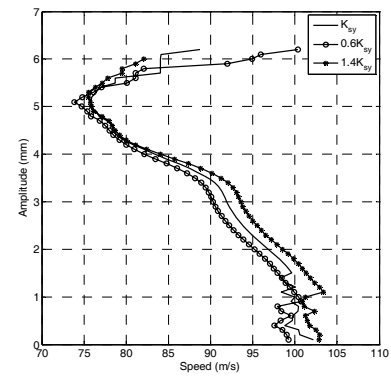

(a)

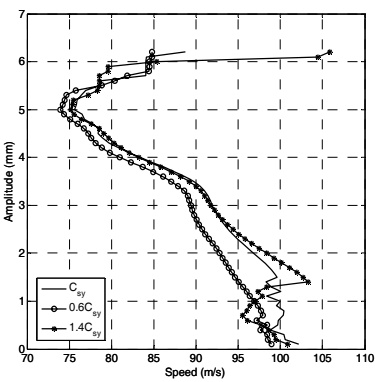

(b)
Fig. 6. Bifurcation analysis results along the change of secondary suspension (a) lateral stiffness; (b) lateral damping.

pension is already sufficiently high and the nonlinear critical speed is almost saturated. The linear critical speed of Case 2 is similar to that of Case 1 but the nonlinear critical speed and its limit cycle amplitude is lower than Case 1. Compared to the decrease rate of the stiffness, the decrease of the nonlinear critical speed is also smaller than expected. These aspects can be also observed in Cases 4 and 5 (Fig. 5(b)). However, the bifurcation diagram draws some different shape in the small amplitude region, and the amplitude of the limit cycle at the nonlinear critical speed in Case 4 is similar to those of Case 1 and 5.

The influences of the secondary suspension on the critical speeds are relatively lower than the primary suspension as shown in Fig. 6. The linear critical speed of Case 7 is a little higher than in Case 1, but the nonlinear critical speeds of them are very similar. For Case 6 , the linear and nonlinear critical speeds are slightly smaller than Case 1 . The amplitudes of the limit cycle at the nonlinear critical speed are a bit increased in Cases 6 and 7.

Increasing the stiffness values, the nonlinear critical speeds were increased as shown in Cases 2 7. However, the bifurcation diagrams for the change of the lateral damping coefficient show a different appearance against other cases. The highest nonlinear critical speed is observed not in Case 9 (high damping) but in Case 1 (middle damping). In other words, when high damping is applied, the nonlinear critical speed is rather decreased even though the decreasing amount is very small. The lowest nonlinear critical speed still exists in the low
Table 3. Analysis results for variation of suspension parameters.

\begin{tabular}{c|c|c|c}
\hline & $V_{0}(\mathrm{~m} / \mathrm{s})$ & $V_{n}(\mathrm{~m} / \mathrm{s})$ & $\frac{V_{n}}{V_{0}}(\%)$ \\
\hline Nominal values (Case 1) & 100.723 & 75.614 & 75.07 \\
60\% Kpx (Case 2) & 99.932 & 70.922 & 70.97 \\
140\% Kpx (Case 3) & 96.163 & 76.839 & 79.90 \\
$60 \%$ Kpy (Case 4) & 97.778 & 71.000 & 72.61 \\
140\% Kpy (Case 5) & 100.379 & 77.524 & 77.23 \\
60\% Ksy (Case 6) & 98.823 & 73.886 & 74.77 \\
140\%Ksy (Case 7) & 102.688 & 75.645 & 73.66 \\
60\%Csy (Case 8) & 97.132 & 73.908 & 76.09 \\
140\%Csy (Case 9) & 101.155 & 75.167 & 74.31 \\
\hline
\end{tabular}

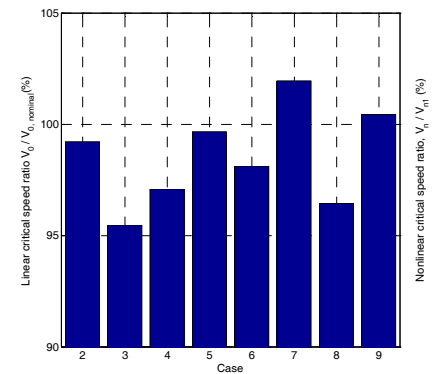

(a)

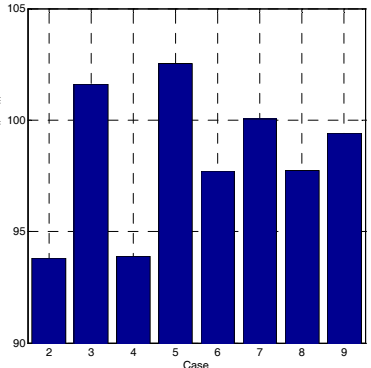

(b)
Fig. 7. Critical speeds ratio for each case (a) linear critical speed ratio; (b) nonlinear critical speed ratio, $V_{n 1}$ is the nonlinear critical speed of Case 1.

damping (Case 8). Table 3 and Fig. 7 summarize the results of the critical speed analysis in this section.

\subsubsection{Wheel/rail friction coefficient}

The critical speed is known that it decreases at increasing friction coefficient between wheel and rail $[1,12]$. But it is also reported that the sensitivity to the friction coefficient might depend on the wheel/rail contact geometry [12]. To estimate the effects of the friction coefficient for the given contact geometry, bifurcation analysis is carried out when the friction coefficient is $0.3,0.4,0.6$. Here, the nominal value is 0.3 . In the case of the given wheel/rail contact geometry, the influence of the friction coefficient on the nonlinear critical speed can be estimated to be low as shown in Table 4 and Fig. 8.

\section{Excitation conditions}

In section 3, we analyzed the nonlinear stability of the given vehicle model and wheel/rail contact geometry and investigated the parametric study for the influences of some model parameters on the linear and nonlinear critical speeds. As stated earlier, the prediction of the nonlinear critical speed is more important than that of the linear critical speed because the hunting phenomenon appears when the speed becomes higher than the nonlinear critical speed, and it has very detrimental effects on the safety of a railway vehicle. However, although the vehicle speed is higher than the nonlinear critical 
Table 4. Analytical results for variation of friction coefficient.

\begin{tabular}{c|c|c}
\hline & $V_{0}(\mathrm{~m} / \mathrm{s})$ & $V_{n}(\mathrm{~m} / \mathrm{s})$ \\
\hline$\mu=0.3$ & 100.723 & 75.614 \\
$\mu=0.4$ & 101.973 & 76.265 \\
$\mu=0.6$ & 102.826 & 76.270 \\
\hline
\end{tabular}

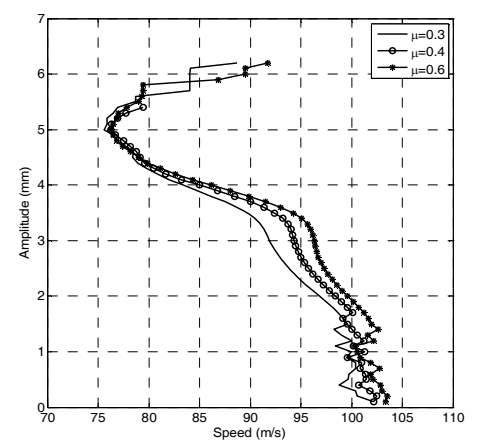

Fig. 8. Bifurcation analysis results for friction coefficients.

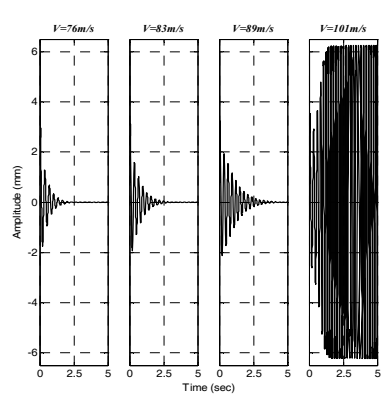

(a)

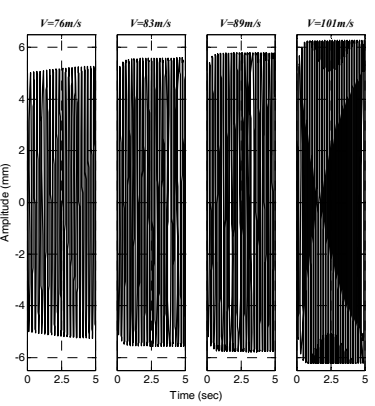

(b)
Fig. 9. Simulation of lateral displacement for first wheelset with following initial conditions (a) $\mathrm{y}_{\mathrm{wj}}(0)=6 \mathrm{~mm}(j=1, \cdots, 4)$, others are zero; (b) all state variables have non-zero initial conditions according to their Poincaré maps.

speed, the hunting cannot always be observed. For example, Fig. 9(a) shows the time response of the investigated vehicle model for certain initial conditions. Limit cycle cannot be seen until the speed becomes $101 \mathrm{~m} / \mathrm{s}$. But in other initial conditions, limit cycle can be observed at the speed of $76 \mathrm{~m} / \mathrm{s}$ as predicted from the bifurcation analysis (Fig. 9(b)). Hence, it is also important to investigate which one of the multiple stable solutions (stationary and stable limit cycle) will be realized depending on initial or disturbance conditions. Particularly, this investigation can give us very useful information when we test a railway vehicle with roller rig test stands.

The general method using the roller rig is to increase the test speed until the linear critical speed appears and start to decrease the test speed slowly when wheelsets have large oscillations. Then the amplitude of the oscillation is gradually reduced and finally becomes zero. Here, the test speed when the oscillation disappears is defined as the nonlinear critical speed. Therefore, to test a vehicle using the roller rig, the maximum allowable speed of the roller rig should be higher than the linear critical speed of test vehicle. But the linear
Table 5. Phases of the sinusoidal track irregularity to each wheelset.

\begin{tabular}{c|c|c|c|c}
\hline Case & 1st wheelset & 2nd wheelset & 3rd wheelset & 4th wheelset \\
\hline E1 & $0^{\circ}$ & $0^{\circ}$ & $0^{\circ}$ & $0^{\circ}$ \\
E2 & $0^{\circ}$ & $0^{\circ}$ & $180^{\circ}$ & $180^{\circ}$ \\
E3 & $0^{\circ}$ & $180^{\circ}$ & $0^{\circ}$ & $180^{\circ}$ \\
E4 & $0^{\circ}$ & $180^{\circ}$ & $180^{\circ}$ & $0^{\circ}$ \\
\hline
\end{tabular}

critical speed of high speed railway vehicle is often higher than the maximum speed of roller rig. In this case, we have to generate the oscillation of wheelsets artificially and check when the oscillation does not disappear with increasing the test speed.

There are two methods to excite the wheelset in the roller rig test: One is to use measured irregularities of real track, and the other is to use a sine wave with constant amplitude and frequency. A sine wave excitation can be used when the grade of the measured track irregularities is not applicable to test vehicle or continuous large amplitude excitation has to be applied. According to the vehicle characteristics and test types, the proper excitation method is selected.

Wagner [24] investigated the domains of attraction and probabilities of occurrence of the stable solutions using a constrained single wheelset model by primary suspension. He expressed the domains of attraction for several initial conditions on the $y-\dot{y}$ and $\psi-\dot{\psi}$ map. But, it is very difficult and complex to apply his expression technique in the full vehicle model because its state variables are so many that the number of cases to be investigated is enormous. Therefore, considering the excitation method of the roller rig test, instead of his expression, excitation conditions are investigated in this section. To achieve this, rails are assumed to have a sine wave irregularity and excite each wheelset during two cycles. Lateral irregularities of the rails can be simulated by changing the contact points according to the relative displacement of wheel and rail. Therefore, the label of abscissas in Fig. 3 implies the relative lateral displacement of wheel and rail. Time response of the first wheelset is simulated with change of amplitude (1 6 mm) and frequency $(1 \sim 5 \mathrm{~Hz})$ of the sine irregularity. The effect of excitation direction is also studied with changing phases of the sinusoidal track irregularity applied to each wheelset. Table 5 expresses the phases of each case.

Fig. 10 shows the excitation conditions for the speed of $76 \mathrm{~m} / \mathrm{s}$. Here, the area of black solid markers denotes the excitation condition of the stationary solution and blank area (white area) is the excitation condition of the stable limit cycle solution. As displayed in the figure, wheelsets should be excited with proper amplitude and frequency so that hunting can be observed. Particularly, excitation direction is estimated to be very important. The excitations of two wheelsets should be opposite direction in the same bogie in order to generate hunting easily. The hunting phenomenon appears even at the low amplitudes as shown in Fig. 10(c) and (d).

We can see those aspects even at higher speed as shown in Figs. 11 and 12. They show the excitation conditions for the 


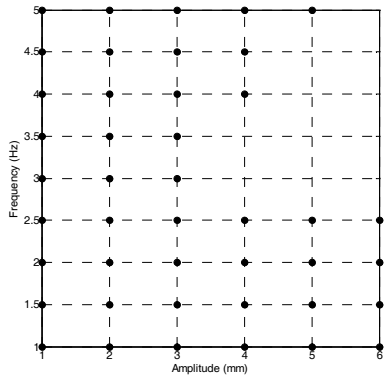

(a)

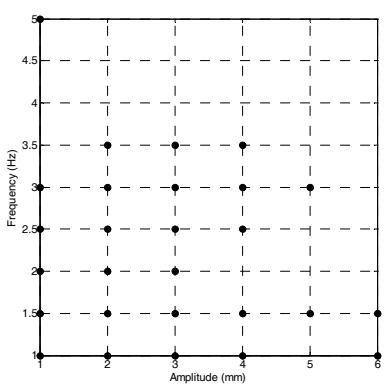

(c)

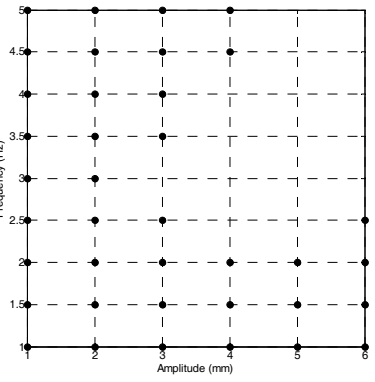

(b)

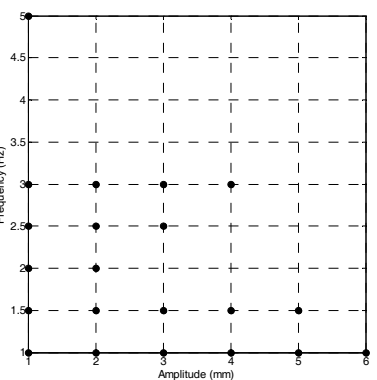

(d)

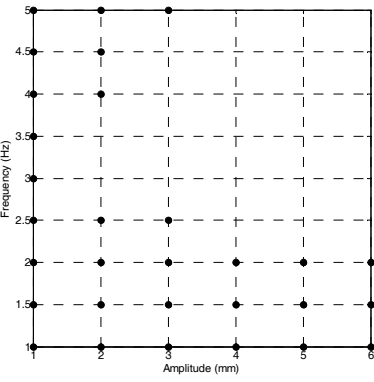

(a)

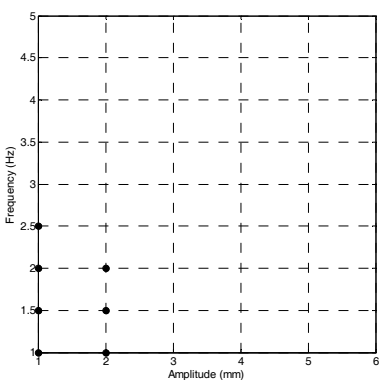

(c)

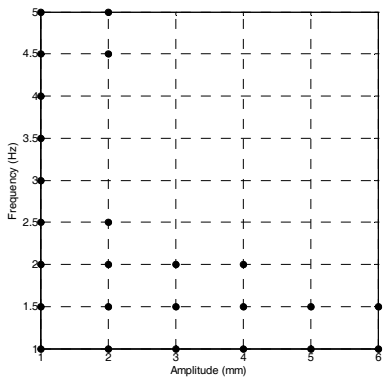

(b)

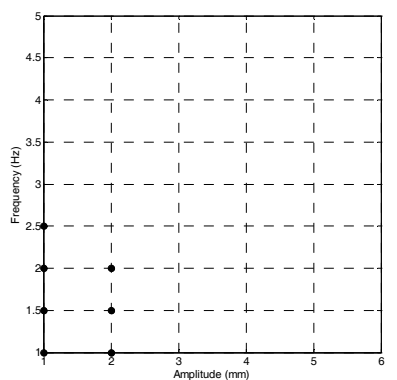

(d)

Fig. 10. Excitation conditions of the first wheelset at speed of $76 \mathrm{~m} / \mathrm{s}$ (a) case E1; (b) case E2; (c) case E3; (d) case E4.

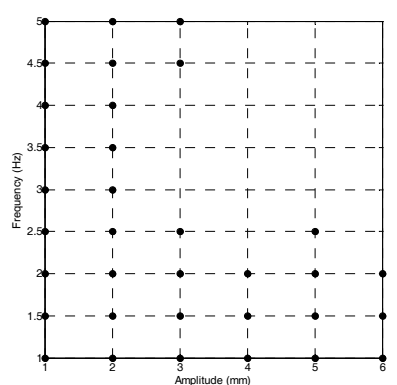

(a)

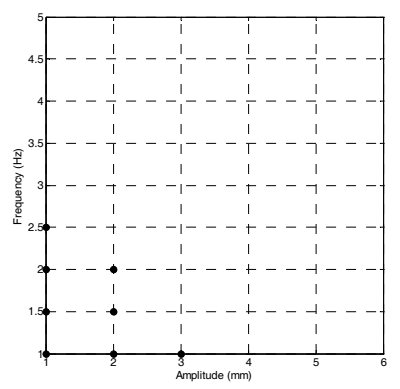

(c)

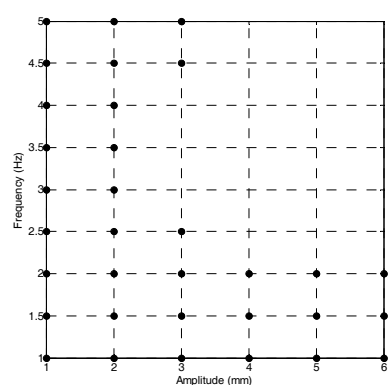

(b)

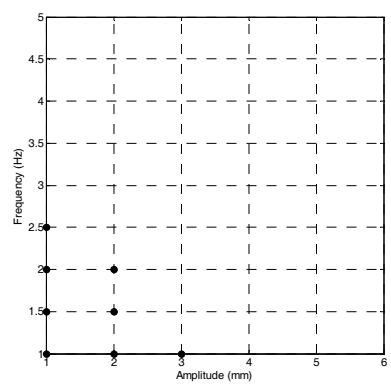

(d)

Fig. 11. Excitation conditions of the first wheelset at speed of $83 \mathrm{~m} / \mathrm{s}$ (a) case E1; (b) case E2; (c) case E3; (d) case E4.

speeds of $83 \mathrm{~m} / \mathrm{s}$ and $89 \mathrm{~m} / \mathrm{s}$, respectively. As expected, the probability of occurrence of the limit cycle (hunting) is increased as the test speed is increased, independently of excitation directions. However, the probability of occurrence of the stationary solution is still a bit high when the excitations of

Fig. 12. Excitation conditions of the first wheelset at speed of $89 \mathrm{~m} / \mathrm{s}$ (a) case E1; (b) case E2; (c) case E3; (d) case E4.

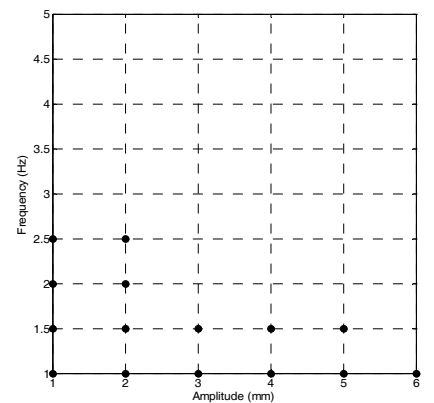

Fig. 13. Excitation conditions of the first wheelset at speed of $101 \mathrm{~m} / \mathrm{s}$ for case E1.

two wheelsets have the same direction in the same bogie. Especially, if all the wheelsets are excited in the same direction such as case E1, a limit cycle may not be observed even at the speed close to the linear critical speed. Fig. 13 shows the excitation conditions for the case E1 at the speed of $101 \mathrm{~m} / \mathrm{s}$. There still exists the region of stationary solution. Therefore, when the sine wave excitation is carried out in the roller rig test or time response analysis, the excitation directions of two wheelsets in the same bogie should be selected to be opposite first and, next, proper amplitude and frequency have to be chosen.

\section{Conclusion}

This paper describes the nonlinear stability of a railway vehicle using bifurcation analysis. In the case of the investigated vehicle model, the influences of the suspension parameters on 
the critical speeds are less than expected. This implies that the suspension parameters are so high that their effects are not severe within the given range and represent indirectly that the critical speeds are more sensitive to wheel/rail contact characteristics than suspension parameters. Friction coefficient has not large effects on the critical speeds for the given model.

Domains of attractions can give us useful information to understand the nonlinear dynamic behavior of a railway vehicle, particularly to test the vehicle with roller rig test stands. When the wheelsets should be excited artificially to find the nonlinear critical speed using sine wave excitation on the roller rig test or time response analysis, it is strongly recommended that two wheelsets in the same bogie have to be excited in an opposite direction. Also, proper amplitude and frequency should be selected to find the nonlinear critical speed accurately. Measured irregularities of track for excited wheelsets should also be carefully selected since they might not be suitable in some cases such as too short applying time, mismatches between the track irregularity grade and test vehicle and so on. Hence, a theoretical analysis of the nonlinear critical speed and excitation conditions has to precede the test.

It should be noted that most of the results of this paper are limited for the investigated vehicle model because they depend heavily on nonlinear parameters like wheel/rail contact geometry, nonlinear suspension parameters (although they were assumed to be linear in this study) and so on. Therefore, in future studies, the nonlinear stabilities and their characteristics of various vehicle models will be investigated and compared with the test results of roller rig. Sophisticated excitation conditions will be also studied in order to acquire more accurate nonlinear critical speed from the roller rig test.

\section{Nomenclature}

a : Longitudinal semi-axis of contact ellipse

$b \quad$ : Lateral semi-axis of contact ellipse

$C_{p x} \quad$ : Longitudinal damping of the primary suspension

$C_{p y} \quad$ : Lateral damping of the primary suspension

$C_{s x}$ : Longitudinal damping of the secondary suspension

$C_{s y} \quad$ : Lateral damping of the secondary suspension

$F_{v j j}, F_{v r j}$ : Lateral creep forces of the left/right wheel at the jth wheelset in the reference frame

$I_{b z} \quad$ : Moment of inertia of the bogie for z-axis

$I_{v} \quad:$ Moment of inertia of the car body for z-axis

$I_{w z}, I_{w y}$ : Moment of inertia of the wheelset for $\mathrm{z}$ and $\mathrm{y}$ axis, respectively

$K_{p x} \quad$ : Longitudinal stiffness of the primary suspension

$K_{p y} \quad$ : Lateral stiffness of the primary suspension

$K_{s x} \quad$ : Longitudinal stiffness of the secondary suspension

$K_{s y} \quad:$ Lateral stiffness of the secondary suspension

$l_{b a} \quad:$ Half of secondary suspension arm in a same bogie

$l_{b b} \quad:$ Half of two center pivots

$l_{w a} \quad$ : Half of primary suspension arm in a same wheelset

$l_{w b} \quad:$ Half of wheel base in a same bogie

$m_{w} \quad:$ Wheelset mass $m_{b} \quad:$ Bogie mass

$m_{v} \quad$ : Car body mass

$N_{y j j}, N_{y r j}$ : Normal forces of the left/right wheel at the jth wheelset in the reference frame

$r_{0} \quad$ : Nominal wheelset rolling radius

$R_{x x j}, R_{x l j}:$ x-axis component of contact point position vector for jth right and left wheel in the reference frame, respectively

$R_{y j j}, R_{y j j}$ : y-axis component of contact point position vector for jth right and left wheel in the reference frame, respectively

$V \quad$ : Traveling velocity of a vehicle

$V_{0 k} \quad$ : Linear critical speed of linear Kalker model

$V_{0} \quad:$ Linear critical speed of linearized model

$V_{n} \quad$ : Nonlinear critical speed

$y_{b i} \quad:$ Lateral displacement of the ith bogie

$y_{v} \quad$ : Lateral displacement of the car body

$y_{w j} \quad:$ Lateral displacement of the jth wheelset

$\psi_{b i} \quad$ : Angle of attack of the ith bogie

$\psi_{w j} \quad$ : Angle of attack of jth wheelset

\section{References}

[1] S. Iwnicki, Handbook of railway vehicle dynamics, CRC Press, Boca Raton, USA (2006).

[2] K. Knothe and F. Böhm, History of stability of railway and road vehicles, J. Vehicle System Dynamics, 31 (1999) 283323.

[3] H. True, On the theory of nonlinear dynamics and its applications in vehicle system dynamics, J. Vehicle System Dynamics, 31 (1999) 393-421.

[4] G. Schupp, Bifurcation analysis of railway vehicles, Multibody System Dynamics, 15 (2006) 25-50.

[5] C. Kaas-Pertersen, Chaos in a railway bogie, Acta Mechanica, 61 (1986) 89-107.

[6] C. N. Jensen, M. Golubitsky and H. True, Symmetry, generic bifurcations, and mode interaction in nonlinear railway dynamics, Int'l. J. Bifurcation and Chaos, 9 (7) (1999) 13211331.

[7] K. Zboinski and M. Dusza, Self-exciting vibrations and Hopf's bifurcation in non-linear stability analysis of rail vehicles in a curved track, European J. Mechanics A/Solids, 29 (2010) 190-203.

[8] J. Zeng and P. Wu, Stability analysis of high speed railway vehicles, JSME Int'l Journal Series C, 47 (2) (2004) 464-470.

[9] H. True and R. Asmund, The dynamics of railway freight wagon wheelset with dry friction damping, J. Vehicle System Dynamics, 38 (2) (2002) 149-163.

[10] H. Molatefi, M. Hecht and M. H. Kadlvar, Effect of suspension system in the lateral stability of railway freight trucks, Proc. IMechE Part F: J. Rail and Rapid Transit, 221 (2007) 399-407.

[11] O. Polach, Comparability of the non-linear and linearized stability assessment during railway vehicle design, J. Vehicle System Dynamics, 44 supplement (2006) 129-138. 
[12] O. Polach, On non-linear methods of bogie stability assessment using computer simulations, Proc. IMechE Part F: J. Rail and Rapid Transit, 220 (2006) 13-27.

[13] A. A. Shabana, K. E. Zaazaa and H. Sugiyama, Railroad vehicle dynamics: A computational approach, CRC Press, New York, USA (2008).

[14] J. J. Kalker, Three-dimensional elastic bodies in rolling contact, Kluwer Academic Publishers, Dordrecht, the Netherlands (1990).

[15] O. Polach, A fast wheel/rail forces calculation computer code, J. Vehicle System Dynamics, 33 (1999) 728-739.

[16] S. Lee and Y. Cheng, Nonlinear analysis on hunting stability for high-speed railway vehicle trucks on curved tracks, ASME J. Vibration and Acoustics, 127 (2005) 324-332.

[17] V. K. Garg and R. V. Dukkipati, Dynamics of railway vehicle systems, Academic Press, Ontario, Canada (1984).

[18] H. Hur, J. Park, W. You and T. Park, A study on the critical speed of worn wheel profile using a scale model, $J$. Mechanical Science and Technology, 23 (2009) 2790-2800.

[19] J. Zeng, W. H. Zang, H. Y. Dai, X. J. Wu and Z. Y. Shen, Hunting instability and $\mathrm{H} \infty$ controlled stabilizer design for high speed railway passenger car, J. Vehicle System Dynamics, 28 supplement (1998) 655-668.

[20] Y. Yuan, A review of trust region algorithms for optimization, Proceedings of ICIAM99, Edinburgh, UK (1999) 5-9.

\section{Appendix A.1}

\section{Vehicle model parameters}

\begin{tabular}{c|l}
\hline Parameters & \multicolumn{1}{c}{ Value (units) } \\
\hline$m_{w}$ & $1.4($ ton $)$ \\
$I_{w z}, I_{w y}$ & $0.965\left(I_{w z}\right), 0.134\left(I_{w y}\right)\left(\right.$ ton $\left.^{2} \mathrm{~m}^{2}\right)$ \\
$m_{b}$ & $3.8($ ton $)$ \\
$I_{b z}$ & $3.8\left(\right.$ ton $\left.\cdot \mathrm{m}^{2}\right)$ \\
$m_{v}$ & $28($ ton $)$ \\
$I_{v}$ & $900\left(\right.$ ton $\left.\cdot \mathrm{m}^{2}\right)$ \\
$r_{0}$ & $0.43(\mathrm{~m})$ \\
$l_{w a}$ & $1(\mathrm{~m})$ \\
$l_{w b}$ & $1.05(\mathrm{~m})$ \\
$l_{b a}$ & $1.125(\mathrm{~m})$ \\
$l_{b b}$ & $6.9(\mathrm{~m})$ \\
$K_{p x}$ & $6.6(\mathrm{MN} / \mathrm{m})$ \\
$K_{p y}$ & $4.4(\mathrm{MN} / \mathrm{m})$ \\
$C_{p x}$ & 0 \\
$C_{p y}$ & 0 \\
$K_{s x}$ & $0.167(\mathrm{MN} / \mathrm{m})$ \\
$K_{s y}$ & $0.167(\mathrm{MN} / \mathrm{m})$ \\
$C_{s x}$ & 0 \\
$C_{s y}$ & $0.096(\mathrm{MN} \cdot \mathrm{s} / \mathrm{m})$ \\
\hline
\end{tabular}

[21] N. K. Cooperrider, The hunting behavior of conventional railway trucks, ASME J. Eng. Industry, 94 (1972) 752-762.

[22] J. T. Pearson, R. M. Goodall, T. X. Mei and G. Himmelstein, Active stability control strategies for a high speed bogie, Elsevier Control Engineering Practice, 12 (2004) 13811391.

[23] S. Shen, T. X. Mei, R. M. Goodall, J. Pearson and G. Himmelstein, A study of active steering strategies for railway bogie, J. Vehicle System Dynamics, 41 supplement (2004) 282-291.

[24] U. Wagner, Nonlinear dynamic behaviour of a railway wheelset, J. Vehicle System Dynamics, 47 (5) (2009) 627640 .

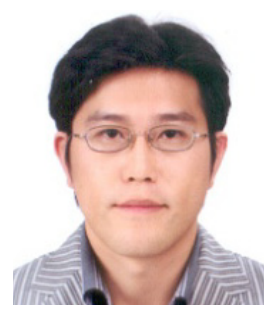

Joon-Hyuk Park received the B.S. degree from KAIST, Daejeon, Korea in 1998, and the M.S. and Ph.D. from Yonsei University, Seoul, Korea in 2000, 2005, respectively, all in Mechanical Engineering. He is a senior researcher at the Vehicle Dynamics \& Propulsion Research Department in Korea Railroad Research Institute. His research interests are related to vehicle dynamics including the mechatronic railway vehicle and running test and evaluation technology for railway vehicles.

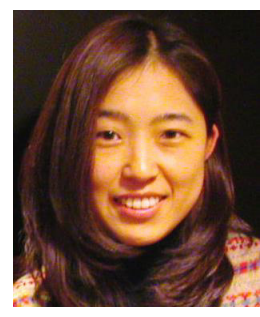

Hyo-In Koh received the M.A. degree in 2001 and the Dr. Ing degree in 2004 from Technical University of Berlin, Germany, in Technical Acoustics. She is a senior researcher at the Railway Environment Research Department in Korea Railroad Research Institute. Her research interests are related to noise reduction and acoustical environment in the railway system.

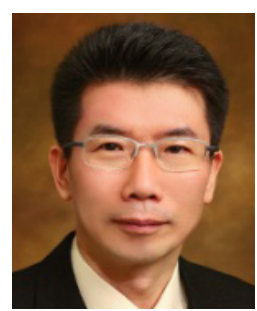

Nam-Po Kim received a B.S. in Mechanical Engineering from Ajou University in 1985. He then went on to receive his M.S. and Ph.D. degrees from Ajou University in 1992 and 2008, respectively. Dr. Kim is currently a principal researcher at the department of vehicle dynamics and propulsion system at Korea Rail Road Research Institute in Uiwang, Korea. His research interests are in the area of railway vehicle dynamics, active control of running gear for railway vehicle and vehicle system engineering. 